\title{
Investigation of spherical and cylindrical catural Iridium targets by photonuclear reaction
}

\author{
Mehmet Emin Korkmaz ${ }^{1, *}$, Osman Agar ${ }^{1}$, and Mustafa Yigit $^{2}$ \\ ${ }^{1}$ Karamanoğlu Mehmetbey University, Faculty of Sciences, Department of Physics, Karaman, Turkey \\ ${ }^{2}$ Aksaray University, Faculty of Arts and Sciences, Department of Physics, Aksaray, Turkey
}

\begin{abstract}
In this study, natural iridium consisting of Ir-191 and Ir-193 isotopes has been irradiated with $21 \mathrm{MeV}$ photons. The distribution of photons, electrons and neutrons fluxes in the spherical and cylindrical natural iridium target have been calculated using MCNPX 2.7.0 Monte Carlo code. The intensity of the photon fluxes on both targets has been compared to the $10^{6}$ particle story by showing them as mesh and optimizing the two targets.
\end{abstract}

\section{Introduction}

Nowadays particle beams are produced by tens of thousands of accelerators in various fields such as hospitals, manufacturing and printing plants, industrial laboratories, ports. More than 350 cyclotrons around world have been producing radioactive isotopes for use in medical applications, e.g. PET scan [1]. The radionuclides have been generally used at nuclear medicine for diagnostic purposes of some diseases as well as in therapy [2,3]. Recently, electron radiotherapy method has been of interest to carry out in the treatment of cancerous cells. The basic component of photonuclear radioisotope production by linear electron accelerators (LINACs) is a bremsstrahlung converter. A high $\mathrm{Z}$ photon converter which is made of tantalum (Ta) or tungsten (W) absorbs the high-energy electrons produced by LINAC. Thereby the energies of electrons are converted into bremsstrahlung photons [4]. On the other hand, nuclear data are needed in many applications which energy related investigations are fusion [5-8], fission, accelerator driven systems, etc. and also nonenergy related investigations are medical radionuclide production [2,3], astrophysics, radiation therapy, etc [9]. Strong beta emitter Ir-192 ( $\mathrm{T}_{1 / 2}=74$ days) has been produced in the wire to use the inner radiotherapy source for prostate cancer treatment. Iridium which is one of the rare platinum group elements on the earth is used for various purposes in medicine and industrial since it's extremely resistant against corrosion and hot. Moreover, there are some application areas such as ignition plug, satellite systems, and industrial monitoring [10-12]. In general, the most stable radioisotope Ir-192 can occur as a consequence of either bombardment of Ir-191 with neutron in a nuclear reactor or separation of a neutron from Ir-193 by photonuclear reaction. In this study, natural iridium targets with two different geometries have irradiated with photon beam of $21 \mathrm{MeV}$ energy produced from a linear accelerator by using Monte Carlo simulations.

\section{Material and Method}

\subsection{Monte Carlo Method and Photonuclear reaction}

Nuclear methods or models are widely needed to estimate nuclear data, especially if the measured data are not available or are unlikely to be produced because of the experimental difficulty $[13,14]$. Monte Carlo is a statistical method. This method is especially useful in complex problems that cannot be solved by deterministic methods [15]. Nowadays the best calculation tool in medical radiation research is the computer programs that use Monte Carlo (MC) methods. MC methods have been used in various radiation therapy branches such as radiation therapy devices, simulations of sources, dose calculations in targets with some geometry. The method is based on the use of random numbers and probability statistics in order to research any problems. Thus, it provides a reasonable approach at higher accuracy in the solution of problems according to the other techniques [16]. Otherwise radiotherapy application of MC method has a disadvantage since it consumes a lot of time. The calculation time depends on the number, energy and type of the produced particle. In order to obtain a good statistical result in calculations, millions of particles must be traced [17]. Therefore, it needs parallel-working super computers with high calculation power in some MC studies [18]. In this work, all calculations were applied by using MCNPX 2.7.0 code developed by Los Alamos National Laboratory (LANL) [19].

\subsection{Iridium Target System}

In this study, two different iridium targets with spherical and cylindrical geometries were irradiated photon beam of $21 \mathrm{MeV}$ energy and $1 \mathrm{~cm}$ radius. It has been calculated for $10^{6}$ particle histories to decrease the statistical errors in simulations of MCNP-X code. The calculations of MC simulation were completed using

Corresponding author: mkorkmaz@kmu.edu.tr 
Intel Xeon PC workstation of a double processor 2.4 $\mathrm{GHz}$ and $48 \mathrm{~GB}$ memory space. The natural iridium target seen in two dimensions is a sphere which has a radius of $1 \mathrm{~cm}$ and located at origin as given in Fig. 1a.

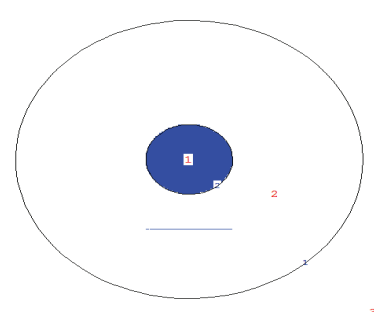

(a)

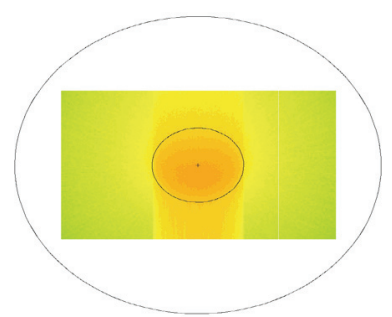

(b)
Fig. 1. (a) The geometry of the spherical natural iridium target (b) The mesh calculations of the photon flux distributions at spherical target

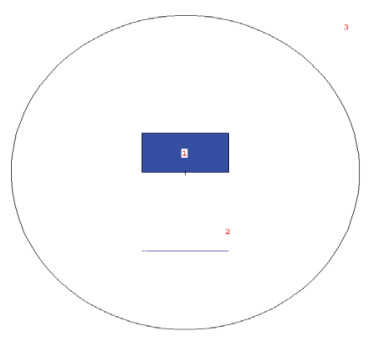

(a)

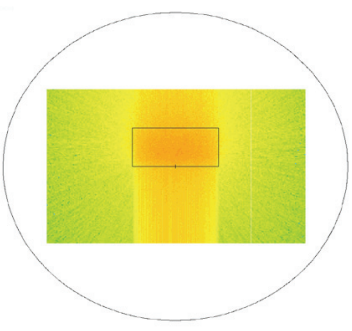

(b)
Fig. 2. (a) The geometry of the cylindrical natural iridium target (b) The mesh calculations of the photon flux distributions at cylindrical target

Photon source is $2 \mathrm{~cm}$ away from the origin. Fig. 1b shows the scattering depending on arrival direction of photon beams for 180000 particle histories on the spherical target and mesh calculations of photon flux density. The photon flux density decreases outward from the center of target. Similarly, the iridium target in Fig. $2 \mathrm{a}$ is a cylindrical form with a radius of $1 \mathrm{~cm}$ and a height of $1 \mathrm{~cm}$. Fig. 2b represents the mesh calculations of photon flux depending on arrival direction of photon beams for 21000 particle histories. The photon flux density in the center of target is higher than the other side.

\section{Results and Discussion}

\subsection{Particle Flux}

Photon, electron and neutron flux distributions of two different natural iridium targets were calculated and compared. The spherical and cylindrical targets were irradiated with photon beam of $21 \mathrm{MeV}$ mono-energetic. Fig. 3 illustrates photon, electron and neutron flux distributions depending on energies for two targets. Although both targets have similar flux distributions, flux of cylindrical are higher than those of spherical target, especially photoneutron flux at $0-13 \mathrm{MeV}$.
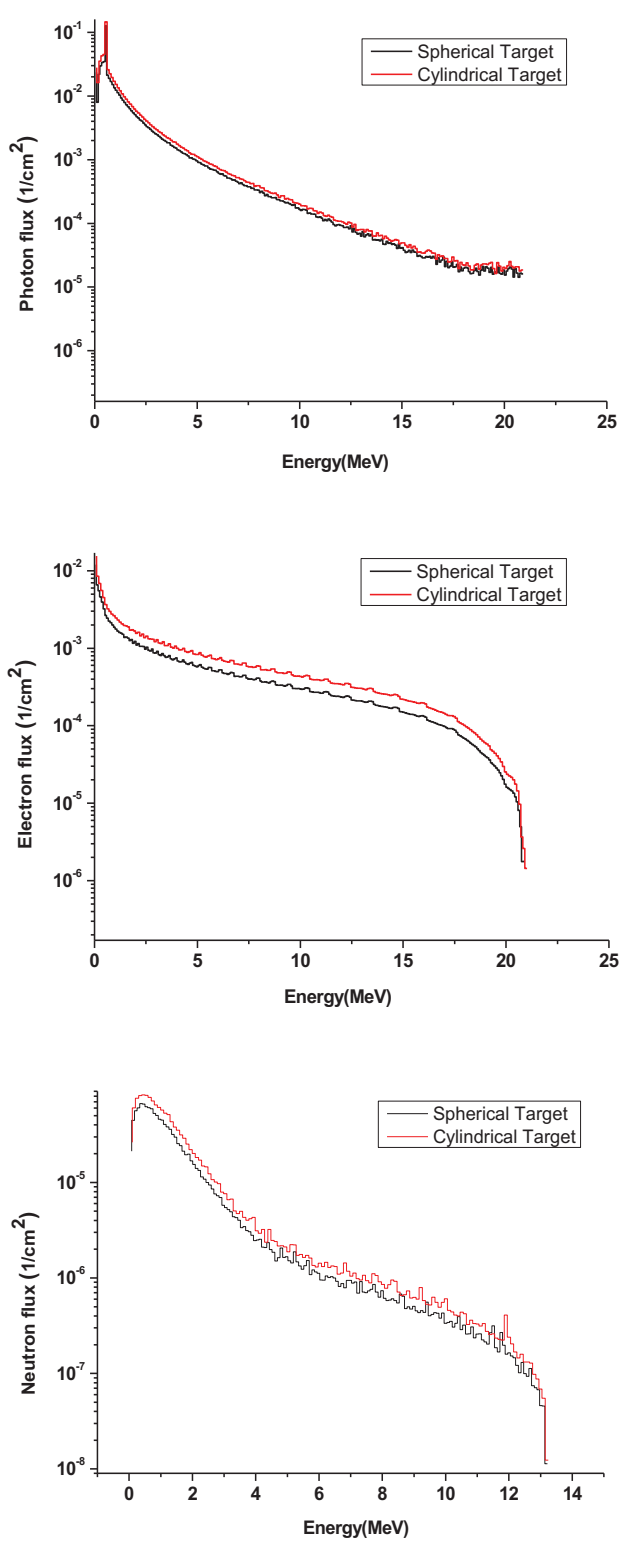

Fig. 3. Photon, electron and neutron flux distributions of spherical and cylindrical targets depending on energies.

\subsection{Energy Deposition}

Fig. 4 represents the stored energies of photon, electron and neutron resulting from nuclear reaction that occur at spherical and cylindrical targets. The energy storage of photons shows approximately similar for different target types as seen in Figure. From the other side, the energy depositions of electrons and neutrons at cylindrical target are quite different and higher than those of spherical. 

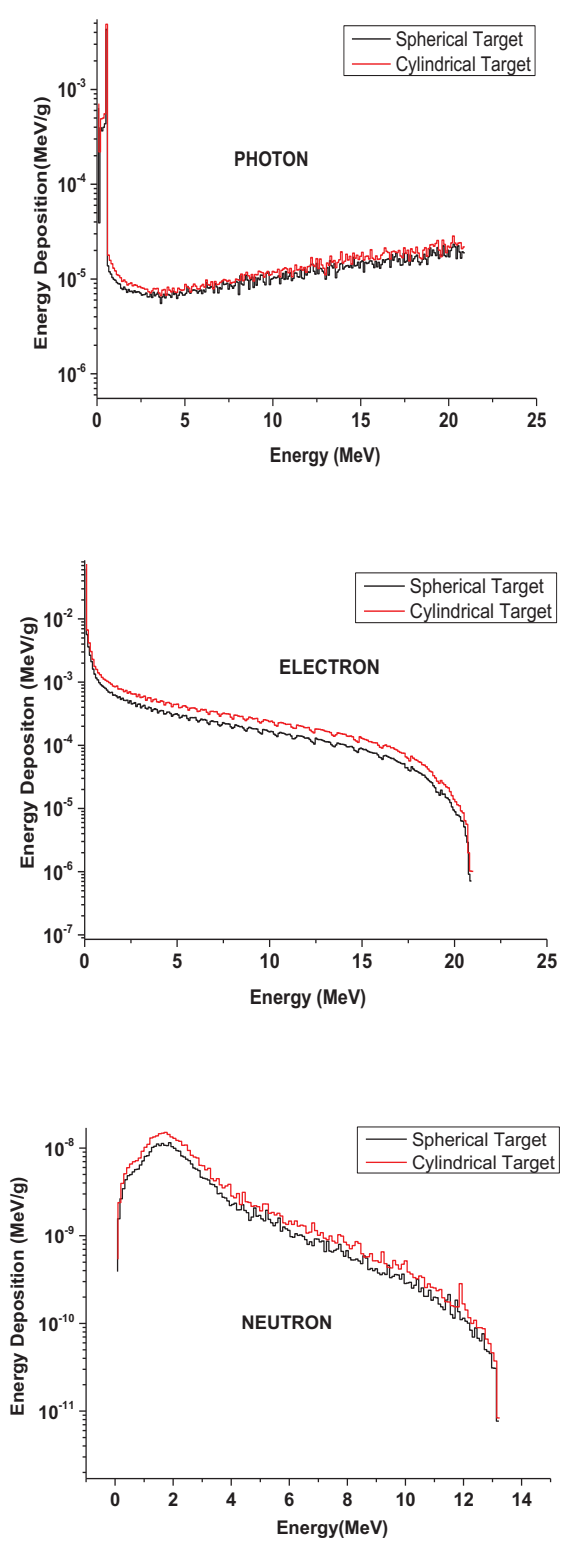

Fig. 4. The energy depositions of photons, electrons and neutrons at spherical and cylindrical targets depending on energies

\section{Conclusions}

The medical electron accelerators are powerful photon sources. Therefore, the photonuclear reaction data can be useful for different technological and scientific developments. One of the most important applications is to produce Ir-192 isotope resulting from photonuclear reaction of Ir-193 stable isotope. Especially, Ir-192 isotope is very important in the treatment of cancer. This isotope has potential to be produced by linear accelerator working at region of 5-25 $\mathrm{MeV}$ energy in hospital. Cylindrical wire-shaped Ir-192 isotope used in brachytherapy is, in general, produced by irradiated with neutrons in reactors. This work shows that sphericalshaped natural iridium target can be produced and give a similar result to those of cylindrical target. Production of medical radioisotopes will increase together with new accelerator technologies. Methods that required R\&D such as beam transmission and targeting for ion therapy will highly be developed.

This study was supported by Karamanoglu Mehmetbey University Scientific Research Project (37-M-16).

\section{References}

1. U.S. Department of Energy Report, Accelerators for America's Future, (2010)

2. A. Kara, T. Korkut, M. Yiğit, E. Tel, Kerntechnik 80, 270 (2015)

3. M. Yiğit and E. Tel, Ann. Nucl. Energy 69, 44 (2014)

4. H. Ghiasi and A. Mesbahi, Iran J. Radiat. Res. 8, 187 (2010)

5. E. Tel, S. Akca, A. Kara, M. Yiğit, A. Aydin, J. Fusion Energ. 32, 531 (2013)

6. H. Sahan, E. Tel, M. Yiğit J. Fusion Energ. 34, 16 (2015)

7. M. Yiğit, E. Tel and İ.H. Sarpün, Nucl. Instrum. Meth. A 385, 59 (2016)

8. H. Korkut, T. Korkut, A. Kara, M. Yiğit, E. Tel, J. Fusion Energ. 35, 591 (2016)

9. M. Yiğit, E. Tel, A. Kara, J. Fusion Energ. 32, 362 (2013)

10. S.S. Belyshev, A.N. Ermankov, B.S. Ishkhanov, V.V. Khankin, A.S. Kurilik, A.A. Kuznetsov, V.I. Shvedunov, K.A. Stopani, Nucl. Instrum. Meth. A 745, 133 (2014)

11. C. Beinke, A. Ben-Shenlomo, M. Abend, M. Port, Radiat. Res. 184, 66 (2015)

12. I. Gerardy, J. Rodenas, S. Gallardo, Appl. Radiat. Isot. 69, 1108 (2011)

13. E. Tel, M. Yiğit, G. Tanir, J. Fusion Energ. 31,184 (2012)

14. E. Tel, M. Yigit, G. Tanir, J. Fusion Energ. 32, 273 (2013)

15. M.E. Korkmaz, M. Yiğit, O. Agar, Kerntechnik 78, 193 (2013)

16. K. Jabbari, A. Sarfehnia, E.B. Podgorsak, J.P. Seuntjens, Phys. Med. Bio. 52, 1171 (2007)

17. M. Zoubair, T. El Bardouni, O. Allaoui, Y. Boulaich, B. El Bakkari, C. El Younoussi, H. Boukhal, E. Chakir, WJNST 3, 14 (2013)

18. T. Liu, X.G. Xu, C.D. Carothers, Ann. Nucl. Energy 82, 230 (2015)

19. D.B. Pelowitz, MCNPX User's Manual, (Version 2.7.0, LA-CP11-00438, 2011) 